\title{
On the general $K$-interpolation method for the sum and the intersection
}

\author{
Irshaad Ahmed ${ }^{*}$ (1) and Muhammad Awais
}

\section{"Correspondence:}

quantized84@yahoo.com

Department of Mathematics,

Government College University,

Faisalabad, Pakistan

\begin{abstract}
Let $\left(A_{0}, A_{1}\right)$ be a compatible couple of normed spaces. We study the interrelation of the general $K$-interpolation spaces of the couple $\left(A_{0}+A_{1}, A_{0} \cap A_{1}\right)$ with those of the couples $\left(A_{0}, A_{1}\right),\left(A_{0}+A_{1}, A_{0}\right),\left(A_{0}+A_{1}, A_{1}\right),\left(A_{0}, A_{0} \cap A_{1}\right)$, and $\left(A_{1}, A_{0} \cap A_{1}\right)$.
\end{abstract}

MSC: $46 \mathrm{~B} 70$

Keywords: sum space; intersection space; $K$-functional; the general $K$-interpolation method; limiting $K$-interpolation methods

\section{Introduction}

Let $\left(A_{0}, A_{1}\right)$ be a compatible couple of normed spaces, i.e. we assume that both $A_{0}$ and $A_{1}$ are continuously embedded in a topological vector space $\mathcal{A}$. The sum of $A_{0}$ and $A_{1}$, denoted by $A_{0}+A_{1}$, is the set of elements $f \in \mathcal{A}$ that can be represented as $f=f_{0}+f_{1}$ where $f_{0} \in A_{0}$ and $f_{1} \in A_{1}$. The norm on the sum space $A_{0}+A_{1}$ is given by

$$
\|f\|_{A_{0}+A_{1}}=\inf \left\{\left\|f_{0}\right\|_{A_{0}}+\left\|f_{1}\right\|_{A_{1}}: f_{0} \in A_{0}, f_{1} \in A_{1}, f=f_{0}+f_{1}\right\} .
$$

The norm on the intersection space $A_{0} \cap A_{1}$ is given by

$$
\|f\|_{A_{0} \cap A_{1}}=\max \left\{\|f\|_{A_{0}},\|f\|_{A_{1}}\right\} .
$$

The Peetre's $K$-functional is defined, for each $f \in A_{0}+A_{1}$ and $t>0$, by

$$
K\left(t, f ; A_{0}, A_{1}\right)=\inf \left\{\left\|f_{0}\right\|_{A_{0}}+t\left\|f_{1}\right\|_{A_{1}}: f_{0} \in A_{0}, f_{1} \in A_{1}, f=f_{0}+f_{1}\right\} .
$$

Let $\Phi$ be a normed space of Lebesgue measurable functions, defined on $(0, \infty)$, with monotone norm: $|g| \leq|h|$ implies $\|g\|_{\Phi} \leq\|h\|_{\Phi}$. Further assume that

$$
t \longmapsto \min \{1, t\} \in \Phi
$$

By definition, the general $K$-interpolation space $\left(A_{0}, A_{1}\right)_{\Phi}$ is a subspace of $A_{0}+A_{1}$ having the following norm:

$$
\|f\|_{\left(A_{0}, A_{1}\right)_{\Phi}}=\left\|K\left(t, f ; A_{0}, A_{1}\right)\right\|_{\Phi} .
$$

(c) The Author(s) 2016. This article is distributed under the terms of the Creative Commons Attribution 4.0 International License (http://creativecommons.org/licenses/by/4.0/), which permits unrestricted use, distribution, and reproduction in any medium, provided you give appropriate credit to the original author(s) and the source, provide a link to the Creative Commons license, and indicate if changes were made. 
Here $\Phi$ is often termed the parameter of the $K$-interpolation method. We refer to [1] for a complete treatment of the general $K$-interpolation method.

Set

$$
\Gamma=([0,1] \times[1, \infty]) \backslash(\{0,1\} \times[1, \infty)) .
$$

Let $(\theta, p) \in \Gamma$, then the classical scale of $K$-interpolation spaces $\left(A_{0}, A_{1}\right)_{\theta, q}$ (see [2] or [3]) is obtained when $\Phi$ is taken to be the weighted Lebesgue space $L_{q}\left(t^{-\theta}\right)$ defined by the norm

$$
\|g\|_{\Phi}= \begin{cases}\left(\int_{0}^{\infty} t^{-\theta}|g(t)|^{p} \frac{d t}{t}\right)^{1 / p}, & (\theta, p) \in(0,1) \times[1, \infty), \\ \sup _{0<t<\infty} t^{-\theta}|g(t)|, & (\theta, p) \in[0,1] \times\{\infty\} .\end{cases}
$$

The following identity was proved by Maligranda [4]:

$$
\left(A_{0}+A_{1}, A_{0} \cap A_{1}\right)_{\theta, p}= \begin{cases}\left(A_{0}, A_{1}\right)_{\theta, p}+\left(A_{0}, A_{1}\right)_{1-\theta, p}, & (\theta, p) \in \Gamma_{1}, \\ \left(A_{0}, A_{1}\right)_{\theta, p} \cap\left(A_{0}, A_{1}\right)_{1-\theta, p}, & (\theta, p) \in \Gamma_{2},\end{cases}
$$

where

$$
\Gamma_{1}=([0,1 / 2) \times[1, \infty]) \backslash(\{0\} \times[1, \infty))
$$

and

$$
\Gamma_{2}=([1 / 2,1] \times[1, \infty]) \backslash(\{1\} \times[1, \infty)) .
$$

Subsequently, Maligranda [5] considered the $K$-interpolation spaces $\left(A_{0}, A_{1}\right)_{\varrho, p}$, which are obtained when $\Phi$ is given by

$$
\|g\|_{\Phi}= \begin{cases}\left(\int_{0}^{\infty}\left(\frac{|g(t)|}{\varrho(t)}\right)^{p} \frac{d t}{t}\right)^{1 / p}, & 1 \leq p<\infty \\ \sup _{0<t<\infty} \frac{|g(t)|}{\varrho(t)}, & p=\infty\end{cases}
$$

and extended the identity (1.2) by imposing certain monotonicity conditions on the parameter function $\varrho$. Another related identity, proved by Persson [6], states that

$$
\left(A_{0}+A_{1}, A_{0} \cap A_{1}\right)_{Q, p}=\left(A_{0}+A_{1}, A_{0}\right)_{Q, p} \cap\left(A_{0}+A_{1}, A_{1}\right)_{Q, p} .
$$

Recently, Haase [7] has completely described how the classical $K$-interpolation spaces for the couples $\left(A_{0}, A_{1}\right),\left(A_{0}+A_{1}, A_{0}\right),\left(A_{0}+A_{1}, A_{1}\right),\left(A_{0}, A_{0} \cap A_{1}\right),\left(A_{1}, A_{0} \cap A_{1}\right)$, and $\left(A_{0}+A_{1}, A_{0} \cap A_{1}\right)$ interrelate. The assertions (1.5)-(1.12) in [7], Theorem 1.1, concern the spaces $\left(A_{0}+A_{1}, A_{0} \cap A_{1}\right)_{\theta, p}$, and the goal of this paper is to extend these assertions by means of replacing the classical scale $\left(A_{0}, A_{1}\right)_{\theta, p}$ by the general scale $\left(A_{0}, A_{1}\right)_{\Phi}$.

The main ingredient of our proofs will be the estimate in Proposition 2.4 (see below) which relates the $K$-functional of the couple $\left(A_{0}+A_{1}, A_{0} \cap A_{1}\right)$ with that of the original couple $\left(A_{0}, A_{1}\right)$, whereas this estimate has not been used in [7]. Consequently, our arguments of the proofs are different from those in [7]. 
We will also apply our general results to the limiting $K$-interpolation spaces $\left(A_{0}, A_{1}\right)_{0, p ; K}$ and $\left(A_{0}, A_{1}\right)_{1, p ; K}$ recently introduced by Cobos, Fernández-Cabrera, and Silvestre [8]. Namely, if the parameter spaces $\Phi_{0}$ and $\Phi_{1}$ are given by the norms

$$
\|g\|_{\Phi_{0}}=\left(\int_{0}^{1}|g(s)|^{p} \frac{d s}{s}\right)^{\frac{1}{p}}+\sup _{s>1}|g(s)|
$$

and

$$
\|g\|_{\Phi_{1}}=\sup _{0<s<1} \frac{|g(s)|}{s}+\left(\int_{1}^{\infty}\left(\frac{|g(s)|}{s}\right)^{p} \frac{d s}{s}\right)^{\frac{1}{p}}
$$

where $1 \leq p<\infty$, then $\left(A_{0}, A_{1}\right)_{\Phi_{0}}=\left(A_{0}, A_{1}\right)_{0, p ; K}$ and $\left(A_{0}, A_{1}\right)_{\Phi_{1}}=\left(A_{0}, A_{1}\right)_{1, p ; K}$. Note that, for limiting values $\theta=0,1$, the space $\left(A_{0}, A_{1}\right)_{\theta, p}$ is trivial (containing only zero element) when $p$ is finite. The space $\left(A_{0}, A_{1}\right)_{0, p ; K}$ corresponds to the limiting value $\theta=0$, and the space $\left(A_{0}, A_{1}\right)_{1, p ; K}$ corresponds to the limiting value $\theta=1$. We will, for convenience, write $\left(A_{0}, A_{1}\right)_{\{0\}, p}$ for $\left(A_{0}, A_{1}\right)_{0, p ; K}$, and $\left(A_{0}, A_{1}\right)_{\{1\}, p}$ for $\left(A_{0}, A_{1}\right)_{1, p ; K}$.

The paper is organised as follows. In Section 2, we establish all necessary background material, whereas Section 3 contains the main results.

\section{Background material}

In the following we will use the notation $A \lesssim B$ for non-negative quantities to mean that $A \leq c B$ for some positive constant $c$ which is independent of appropriate parameters involved in $A$ and $B$. If $A \lesssim B$ and $B \lesssim A$, we will write $A \approx B$. Moreover, we will use the symbol $X \hookrightarrow Y$ to show that $X$ is continuously embedded in $Y$.

The elementary but useful properties of the $K$-functional are collected in the following proposition.

Proposition $2.1([3])$ Let $\left(A_{0}, A_{1}\right)$ be a compatible couple of normed spaces. Then $K(t, f$; $\left.A_{0}, A_{1}\right)$ is non-decreasing in $t$, and $K\left(t, f ; A_{0}, A_{1}\right) / t$ is non-increasing in $t$. Moreover, we have

$$
\begin{aligned}
& K\left(t, f ; A_{0}, A_{1}\right) \leq\|f\|_{A_{0}}, \quad f \in A_{0}, t>0 ; \\
& K\left(t, f ; A_{0}, A_{1}\right) \leq t\|f\|_{A_{1}}, \quad f \in A_{1}, t>0 ; \\
& K\left(t, f ; A_{0}, A_{1}\right)=t K\left(t^{-1}, f ; A_{1}, A_{0}\right), \quad f \in A_{0}+A_{1}, t>0 ; \\
& K\left(t, f+g ; A_{0}, A_{1}\right) \leq K\left(t, f ; A_{0}, A_{1}\right)+K\left(t, g ; A_{0}, A_{1}\right), \quad f, g \in A_{0}+A_{1}, t>0 .
\end{aligned}
$$

In the next three propositions, we describe some formulas which relate the $K$-functional of the couples $\left(A_{0}+A_{1}, A_{1}\right),\left(A_{0}, A_{0} \cap A_{1}\right)$, and $\left(A_{0}+A_{1}, A_{0} \cap A_{1}\right)$ with that of the original couple $\left(A_{0}, A_{1}\right)$.

Proposition 2.2 Let $\left(A_{0}, A_{1}\right)$ be a compatible couple of normed spaces, and let $f \in A_{0}+A_{1}$. Then

$$
K\left(t, f ; A_{0}+A_{1}, A_{1}\right)=K\left(t, f ; A_{0}, A_{1}\right), \quad 0<t<1 .
$$


Proof In view of (2.3), the proof follows immediately from the following relation:

$$
K\left(t, f ; A_{0}, A_{0}+A_{1}\right)=K\left(t, f ; A_{0}, A_{1}\right), \quad t>1,
$$

which has been derived in [7], Lemma 2.1.

For the proof of the next result, we refer to [7], Lemma 2.3.

Proposition 2.3 Let $\left(A_{0}, A_{1}\right)$ be a compatible couple of normed spaces, and let $f \in A_{0}$. Then

$$
K\left(t, f ; A_{0}, A_{0} \cap A_{1}\right) \lesssim K\left(t, f ; A_{0}, A_{1}\right)+t\|f\|_{A_{0}}, \quad 0<t<1 .
$$

The next result is derived in [4], Theorem 3.

Proposition 2.4 Let $\left(A_{0}, A_{1}\right)$ be a compatible couple of normed space, and let $f \in A_{0}+A_{1}$. Then

$$
K\left(t, f ; A_{0}+A_{1}, A_{0} \cap A_{1}\right) \approx K\left(t, f ; A_{0}, A_{1}\right)+t K\left(t^{-1}, f ; A_{0}, A_{1}\right), \quad 0<t<1 .
$$

In our proofs, we will make use of the fact that, for a parameter space $\Phi$, both $\left\|s \chi_{(0,1)}(s)\right\|_{\Phi}$ and $\left\|\chi_{(1, \infty)}\right\|_{\Phi}$ are finite. This fact is a simple consequence of (1.1). Moreover, in view of the monotonicity of the norm $\|\cdot\|_{\Phi}$ and the fact that $K\left(t, f ; A_{0}, A_{1}\right)=\|f\|_{A_{0}+A_{1}}$, we have

$$
\|f\|_{\left(A_{0}, A_{1}\right)_{\Phi}} \approx\left\|\chi_{(0,1)}(t) K\left(t, f ; A_{0}, A_{1}\right)\right\|_{\Phi}+\left\|\chi_{(1, \infty)}(t) K\left(t, f ; A_{0}, A_{1}\right)\right\|_{\Phi} .
$$

We will make use of the next result, without explicitly mentioning it, in our proofs.

Proposition 2.5 Let $\left(A_{0}, A_{1}\right)$ be a compatible couple of normed spaces, and assume that $A_{1} \hookrightarrow A_{0}$. Then

$$
\|f\|_{\left(A_{0}, A_{1}\right)_{\Phi}} \approx\left\|\chi_{(0,1)}(s) K\left(s, f ; A_{0}, A_{1}\right)\right\|_{\Phi} .
$$

Proof It will suffice to derive

$$
\|f\|_{\left(A_{0}, A_{1}\right)_{\Phi}} \lesssim\left\|\chi_{(0,1)}(s) K\left(s, f ; A_{0}, A_{1}\right)\right\|_{\Phi}
$$

as the converse estimate is trivial. Using (2.6) and (2.1), we get

$$
\begin{aligned}
\|f\|_{\left(A_{0}, A_{1}\right)_{\Phi}} & \lesssim\left\|\chi_{(0,1)}(s) K\left(s, f ; A_{0}, A_{1}\right)\right\|_{\Phi}+\|f\|_{A_{1}}\left\|\chi_{(1, \infty)}\right\|_{\Phi} \\
& \approx\left\|\chi_{(0,1)}(s) K\left(s, f ; A_{0}, A_{1}\right)\right\|_{\Phi}+\|f\|_{A_{1}},
\end{aligned}
$$

as our assumption $A_{1} \hookrightarrow A_{0}$ implies that $\|f\|_{A_{0}} \approx\|f\|_{A_{0}+A_{1}}$, so

$$
\|f\|_{\left(A_{0}, A_{1}\right)_{\Phi}} \lesssim\left\|\chi_{(0,1)}(s) K\left(s, f ; A_{0}, A_{1}\right)\right\|_{\Phi}+\|f\|_{A_{0}+A_{1}} .
$$


Since $K\left(t, f ; A_{0}, A_{1}\right) / t$ is non-increasing in $t$, we obtain

$$
\left\|\chi_{(0,1)}(s) K\left(s, f ; A_{0}, A_{1}\right)\right\|_{\Phi} \geq K\left(1, f ; A_{0}, A_{1}\right)\left\|s \chi_{(0,1)(s)}\right\|_{\Phi},
$$

which gives

$$
\|f\|_{A_{0}+A_{1}} \lesssim\left\|\chi_{(0,1)}(s) K\left(s, f ; A_{0}, A_{1}\right)\right\|_{\Phi} .
$$

Now (2.7) follows from (2.8) and (2.9). The proof is complete.

\section{Main results}

Theorem 3.1 Let $\left(A_{0}, A_{1}\right)$ be a compatible couple of normed spaces. Then, for an arbitrary parameter space $\Phi$, we have with equivalent norms

$$
\left(A_{0}+A_{1}, A_{0}\right)_{\Phi} \cap\left(A_{0}+A_{1}, A_{1}\right)_{\Phi}=\left(A_{0}+A_{1}, A_{0} \cap A_{1}\right)_{\Phi}
$$

Proof Put $B_{0}=\left(A_{0}+A_{1}, A_{0}\right)_{\Phi}, B_{1}=\left(A_{0}+A_{1}, A_{1}\right)_{\Phi}$, and $B=\left(A_{0}+A_{1}, A_{0} \cap A_{1}\right)_{\Phi}$. Let $f \in$ $A_{0}+A_{1}$. Then by Proposition 2.4

$$
\|f\|_{B} \approx\left\|\chi_{(0,1)}(s) K\left(s, f ; A_{0}, A_{1}\right)\right\|_{\Phi}+\left\|\chi_{(0,1)}(s) s K\left(s^{-1}, f ; A_{0}, A_{1}\right)\right\|_{\Phi}
$$

next making use of (2.3), we arrive at

$$
\|f\|_{B} \approx\left\|\chi_{(0,1)}(s) K\left(s, f ; A_{0}, A_{1}\right)\right\|_{\Phi}+\left\|\chi_{(0,1)}(s) K\left(s, f ; A_{1}, A_{0}\right)\right\|_{\Phi} .
$$

Finally, appealing to Proposition 2.2, we get

$$
\|f\|_{B} \approx\|f\|_{B_{0}}+\|f\|_{B_{1}}
$$

which concludes the proof.

Remark 3.2 The result of Theorem 3.1 generalizes the assertion (1.5) in [7], Theorem 1.1.

Theorem 3.3 Let $\left(A_{0}, A_{1}\right)$ be a compatible couple of normed spaces. Then, for an arbitrary parameter space $\Phi$, we have with equivalent norms

$$
\left(A_{0}, A_{0} \cap A_{1}\right)_{\Phi}+\left(A_{1}, A_{0} \cap A_{1}\right)_{\Phi}=\left(A_{0}+A_{1}, A_{0} \cap A_{1}\right)_{\Phi}
$$

Proof Put $B_{0}=\left(A_{0}, A_{0} \cap A_{1}\right)_{\Phi}, B_{1}=\left(A_{1}, A_{0} \cap A_{1}\right)_{\Phi}$ and $B=\left(A_{0}+A_{1}, A_{0} \cap A_{1}\right)_{\Phi}$. Let $f \in$ $B_{0}+B_{1}$, and take an arbitrary decomposition $f=f_{0}+f_{1}$ with $f_{0} \in B_{0}$ and $f_{1} \in B_{1}$. Then by (2.4), we have

$$
\begin{aligned}
\|f\|_{B} \lesssim & \left\|\chi_{(0,1)}(s) K\left(s, f_{0} ; A_{0}+A_{1}, A_{0} \cap A_{1}\right)\right\|_{\Phi} \\
& +\left\|\chi_{(0,1)}(s) K\left(s, f_{1} ; A_{0}+A_{1}, A_{0} \cap A_{1}\right)\right\|_{\Phi}
\end{aligned}
$$

now applying the simple fact that

$$
K\left(t, f_{j} ; A_{0}+A_{1}, A_{0} \cap A_{1}\right) \leq K\left(t, f_{j} ; A_{j}, A_{0} \cap A_{1}\right) \quad(j=0,1), t>0,
$$


we obtain

$$
\|f\|_{B} \lesssim\left\|f_{0}\right\|_{B_{0}}+\left\|f_{1}\right\|_{B_{1}}
$$

from which the estimate $\|f\|_{B} \lesssim\|f\|_{B_{0}+B_{1}}$ follows as the decomposition $f=f_{0}+f_{1}$ is arbitrary. In order to establish the converse estimate, we take $f \in B$ and note that there exists (by definition of the norm on $A_{0}+A_{1}$ ) a particular decomposition $f=f_{0}+f_{1}$ with $f_{0} \in A_{0}$ and $f_{1} \in A_{1}$ such that

$$
\left\|f_{0}\right\|_{A_{0}}+\left\|f_{1}\right\|_{A_{1}} \lesssim\|f\|_{A_{0}+A_{1}}
$$

By Proposition 2.3,

$$
\begin{aligned}
\left\|f_{0}\right\|_{B_{0}} & \lesssim\left\|\chi_{(0,1)}(s) K\left(s, f_{0} ; A_{0}, A_{1}\right)\right\|_{\Phi}+\left\|s \chi_{(0,1)}(s)\right\|_{\Phi}\left\|f_{0}\right\|_{A_{0}} \\
& \approx\left\|\chi_{(0,1)}(s) K\left(s, f_{0} ; A_{0}, A_{1}\right)\right\|_{\Phi}+\left\|f_{0}\right\|_{A_{0}},
\end{aligned}
$$

since $f_{0}=f-f_{1}$, we get by $(2.4)$

$$
\left\|f_{0}\right\|_{B_{0}} \lesssim\left\|\chi_{(0,1)}(s) K\left(s, f ; A_{0}, A_{1}\right)\right\|_{\Phi}+\left\|\chi_{(0,1)}(s) K\left(s, f_{1} ; A_{0}, A_{1}\right)\right\|_{\Phi}+\left\|f_{0}\right\|_{A_{0}}
$$

next we use (2.2) to obtain

$$
\begin{aligned}
\left\|f_{0}\right\|_{B_{0}} & \lesssim\left\|\chi_{(0,1)}(s) K\left(s, f ; A_{0}, A_{1}\right)\right\|_{\Phi}+\left\|s \chi_{(0,1)}(s)\right\|_{\Phi}\left\|f_{1}\right\|_{A_{1}}+\left\|f_{0}\right\|_{A_{0}} \\
& \approx\left\|\chi_{(0,1)}(s) K\left(s, f ; A_{0}, A_{1}\right)\right\|_{\Phi}+\left\|f_{1}\right\|_{A_{1}}+\left\|f_{0}\right\|_{A_{0}}
\end{aligned}
$$

and, using (3.1), we get

$$
\left\|f_{0}\right\|_{B_{0}} \lesssim\left\|\chi(0,1)(s) K\left(s, f ; A_{0}, A_{1}\right)\right\|_{\Phi}+\|f\|_{A_{0}+A_{1}}
$$

in accordance with (2.9), we deduce that

$$
\left\|f_{0}\right\|_{B_{0}} \lesssim\left\|\chi_{(0,1)}(s) K\left(s, f ; A_{0}, A_{1}\right)\right\|_{\Phi} .
$$

Analogously, we can obtain

$$
\left\|f_{1}\right\|_{B_{1}} \lesssim\left\|\chi_{(0,1)}(s) s K\left(s^{-1}, f ; A_{0}, A_{1}\right)\right\|_{\Phi} .
$$

Therefore, combining the previous two estimates, we find that

$$
\left\|f_{0}\right\|_{B_{0}}+\left\|f_{1}\right\|_{B_{1}} \lesssim\left\|\chi_{(0,1)}(s) K\left(s, f ; A_{0}, A_{1}\right)\right\|_{\Phi}+\left\|\chi_{(0,1)}(s) s K\left(s^{-1}, f ; A_{0}, A_{1}\right)\right\|_{\Phi},
$$

from which, in view of Proposition 2.4, it follows that

$$
\|f\|_{B_{0}+B_{1}} \lesssim\|f\|_{B}
$$

which completes the proof. 
Remark 3.4 The result of Theorem 3.3 generalizes the assertion (1.6) in [7], Theorem 1.1.

In order to formulate the further results, we need the following conditions on the parameter spaces $\Phi_{0}$ and $\Phi_{1}$ :

$\left(\mathrm{C}_{1}\right)\left\|\chi_{(0,1)}(s) g(s)\right\|_{\Phi_{0}} \lesssim\left\|\chi_{(0,1)}(s) g(s)\right\|_{\Phi_{1}}$.

$\left(C_{2}\right)\left\|\chi_{(0,1)}(s) g(s)\right\|_{\Phi_{1}} \lesssim\left\|\chi_{(0,1)}(s) g(s)\right\|_{\Phi_{0}}$.

$\left(C_{3}\right)\left\|\chi_{(1, \infty)}(s) g(s)\right\|_{\Phi_{1}} \lesssim\left\|\chi_{(1, \infty)}(s) g(s)\right\|_{\Phi_{0}}$.

$\left(\mathrm{C}_{4}\right)\left\|\chi_{(1, \infty)}(s) g(s)\right\|_{\Phi_{0}} \approx\left\|\chi_{(0,1)}(s) s g(1 / s)\right\|_{\Phi_{1}}$.

$\left(C_{5}\right)\left\|\chi_{(1, \infty)}(s) g(s)\right\|_{\Phi_{1}} \approx\left\|\chi_{(0,1)}(s) \operatorname{sg}(1 / s)\right\|_{\Phi_{0}}$.

Remark 3.5 Let $(\theta, p) \in \Gamma$, and assume that $\Phi_{0}$ and $\Phi_{1}$ are given by the norms

$$
\|g\|_{\Phi_{0}}= \begin{cases}\left(\int_{0}^{\infty} t^{-\theta}|g(t)|^{p} \frac{d t}{t}\right)^{1 / p}, & (\theta, p) \in(0,1) \times[1, \infty), \\ \sup _{0<t<\infty} t^{-\theta}|g(t)|, & (\theta, p) \in[0,1] \times\{\infty\},\end{cases}
$$

and

$$
\|g\|_{\Phi_{1}}= \begin{cases}\left(\int_{0}^{\infty} t^{1-\theta}|g(t)|^{p} \frac{d t}{t}\right)^{1 / p}, & (\theta, p) \in(0,1) \times[1, \infty) \\ \sup _{0<t<\infty} t^{1-\theta}|g(t)|, & (\theta, p) \in[0,1] \times\{\infty\}\end{cases}
$$

Then it is easy to see that $\left(C_{1}\right)$ and $\left(C_{3}\right)$ hold for $(\theta, p) \in \Gamma_{1}$, and $\left(C_{2}\right)$ holds for $(\theta, p) \in \Gamma_{2}$. The conditions $\left(\mathrm{C}_{4}\right)$ and $\left(\mathrm{C}_{5}\right)$ hold trivially for all $(\theta, p) \in \Gamma$.

Remark 3.6 Let $1 \leq p<\infty$, and assume that $\Phi_{0}$ and $\Phi_{1}$ are given by (1.3) and (1.4). Then we note that $\left(C_{1}\right),\left(C_{3}\right),\left(C_{4}\right)$, and $\left(C_{5}\right)$ hold.

Theorem 3.7 Let $\left(A_{0}, A_{1}\right)$ be a compatible couple of normed spaces, and assume that the parameter spaces $\Phi_{0}$ and $\Phi_{1}$ satisfy $\left(C_{1}\right),\left(C_{3}\right)$ and $\left(C_{4}\right)$. Then we have with equivalent norms

$$
\left(A_{0}, A_{1}\right)_{\Phi_{0}} \cap\left(A_{0}, A_{1}\right)_{\Phi_{1}}=\left(A_{0}+A_{1}, A_{0} \cap A_{1}\right)_{\Phi_{1}} .
$$

Proof Put $B_{0}=\left(A_{0}, A_{1}\right)_{\Phi_{0}}, B_{1}=\left(A_{0}, A_{1}\right)_{\Phi_{1}}$ and $B=\left(A_{0}+A_{1}, A_{0} \cap A_{1}\right)_{\Phi_{1}}$. Let $f \in A_{0}+A_{1}$. Then

$$
\begin{aligned}
\|f\|_{B_{0}}+\|f\|_{B_{1}} \approx & \left\|\chi_{(0,1)}(s) K\left(s, f ; A_{0}, A_{1}\right)\right\|_{\Phi_{0}}+\left\|\chi_{(1, \infty)}(s) K\left(s, f ; A_{0}, A_{1}\right)\right\|_{\Phi_{0}} \\
& +\left\|\chi_{(0,1)}(s) K\left(s, f ; A_{0}, A_{1}\right)\right\|_{\Phi_{1}}+\left\|\chi_{(1, \infty)}(s) K\left(s, f ; A_{0}, A_{1}\right)\right\|_{\Phi_{1}},
\end{aligned}
$$

which, in view of $\left(\mathrm{C}_{1}\right)$ and $\left(\mathrm{C}_{3}\right)$, reduces to

$$
\|f\|_{B_{0}}+\|f\|_{B_{1}} \approx\left\|\chi_{(0,1)}(s) K\left(s, f ; A_{0}, A_{1}\right)\right\|_{\Phi_{1}}+\left\|\chi_{(1, \infty)}(s) K\left(s, f ; A_{0}, A_{1}\right)\right\|_{\Phi_{0}},
$$

at this point we use $\left(\mathrm{C}_{4}\right)$ to obtain

$$
\|f\|_{B_{0}}+\|f\|_{B_{1}} \approx\left\|\chi_{(0,1)}(s) K\left(s, f ; A_{0}, A_{1}\right)\right\|_{\Phi_{1}}+\left\|\chi_{(0,1)}(s) K\left(s^{-1}, f ; A_{0}, A_{1}\right)\right\|_{\Phi_{1}},
$$


finally, applying Proposition 2.4, we conclude that

$$
\|f\|_{B_{0}}+\|f\|_{B_{1}} \approx\|f\|_{B}
$$

The proof is complete.

Remark 3.8 Applying Theorem 3.7 to the parameter spaces $\Phi_{0}$ and $\Phi_{1}$ given by (3.2) and (3.3), we get back the result (1.7) in [7], Theorem 1.1, for $(\theta, p) \in \Gamma_{1}$. Note that the case when $(\theta, p) \in \Gamma_{2}$ follows from the case when $(\theta, p) \in \Gamma_{1}$ by replacing $\theta$ by $1-\theta$.

Corollary 3.9 Let $\left(A_{0}, A_{1}\right)$ be a compatible couple of normed spaces, and let $1 \leq p<\infty$. Then we have with equivalent norms

$$
\left(A_{0}, A_{1}\right)_{\{0\}, p} \cap\left(A_{0}, A_{1}\right)_{\{1\}, p}=\left(A_{0}+A_{1}, A_{0} \cap A_{1}\right)_{\{1\}, p} .
$$

Proof The proof follows by applying Theorem 3.7 to the parameter spaces $\Phi_{0}$ and $\Phi_{1}$ given by (1.3) and (1.4).

Theorem 3.10 Let $\left(A_{0}, A_{1}\right)$ be a compatible couple of normed spaces, and assume that the parameter spaces $\Phi_{0}$ and $\Phi_{1}$ satisfy $\left(C_{1}\right),\left(C_{3}\right)$, and $\left(C_{5}\right)$. Then we have with equivalent norms

$$
\left(A_{0}, A_{1}\right)_{\Phi_{0}}+\left(A_{0}, A_{1}\right)_{\Phi_{1}}=\left(A_{0}+A_{1}, A_{0} \cap A_{1}\right)_{\Phi_{0}} .
$$

Proof Put $B_{0}=\left(A_{0}, A_{1}\right)_{\Phi_{0}}, B_{1}=\left(A_{0}, A_{1}\right)_{\Phi_{1}}$ and $B=\left(A_{0}+A_{1}, A_{0} \cap A_{1}\right)_{\Phi_{0}}$. Let $f \in B_{0}+B_{1}$, and write $f=f_{0}+f_{1}$, where $f_{0} \in B_{0}$ and $f_{1} \in B_{1}$. Now by Proposition 2.4, we have

$$
\|f\|_{B} \approx\left\|\chi_{(0,1)}(s) K\left(s, f ; A_{0}, A_{1}\right)\right\|_{\Phi_{0}}+\left\|\chi(0,1)(s) s K\left(s^{-1}, f ; A_{0}, A_{1}\right)\right\|_{\Phi_{0}},
$$

using $\left(\mathrm{C}_{5}\right)$ gives

$$
\|f\|_{B} \approx\left\|\chi_{(0,1)}(s) K\left(s, f ; A_{0}, A_{1}\right)\right\|_{\Phi_{0}}+\left\|\chi_{(1, \infty)}(s) K\left(s, f ; A_{0}, A_{1}\right)\right\|_{\Phi_{1}}
$$

since $f=f_{0}+f_{1}$, so by $(2.4)$, we have

$$
\begin{aligned}
\|f\|_{B} \lesssim & \left\|\chi_{(0,1)}(s) K\left(s, f_{0} ; A_{0}, A_{1}\right)\right\|_{\Phi_{0}}+\left\|\chi_{(0,1)}(s) K\left(s, f_{1} ; A_{0}, A_{1}\right)\right\|_{\Phi_{0}} \\
& +\left\|\chi_{(1, \infty)}(s) K\left(s, f_{0} ; A_{0}, A_{1}\right)\right\|_{\Phi_{1}}+\left\|\chi_{(1, \infty)}(s) K\left(s, f_{1} ; A_{0}, A_{1}\right)\right\|_{\Phi_{1}},
\end{aligned}
$$

by $\left(C_{1}\right)$ and $\left(C_{3}\right)$, we arrive at

$$
\begin{aligned}
\|f\|_{B} \lesssim & \left\|\chi_{(0,1)}(s) K\left(s, f_{0} ; A_{0}, A_{1}\right)\right\|_{\Phi_{1}}+\left\|\chi_{(0,1)}(s) K\left(s, f_{1} ; A_{0}, A_{1}\right)\right\|_{\Phi_{0}} \\
& +\left\|\chi_{(1, \infty)}(s) K\left(s, f_{0} ; A_{0}, A_{1}\right)\right\|_{\Phi_{1}}+\left\|\chi_{(1, \infty)}(s) K\left(s, f_{1} ; A_{0}, A_{1}\right)\right\|_{\Phi_{0}},
\end{aligned}
$$

which gives

$$
\|f\|_{B} \lesssim\left\|f_{0}\right\|_{B_{0}}+\left\|f_{1}\right\|_{B_{1}},
$$


from which the estimate $\|f\|_{B} \lesssim\|f\|_{B_{0}+B_{1}}$ follows. To derive the other estimate, take $f \in B$, and choose a particular decomposition $f=f_{0}+f_{1}$, with $f_{0} \in A_{0}$ and $f_{1} \in A_{1}$, satisfying (3.1). Then

$$
\begin{aligned}
\left\|f_{0}\right\|_{B_{0}} & \approx\left\|\chi_{(0,1)}(s) K\left(s, f_{0} ; A_{0}, A_{1}\right)\right\|_{\Phi_{0}}+\left\|\chi_{(1, \infty)}(s) K\left(s, f_{0} ; A_{0}, A_{1}\right)\right\|_{\Phi_{0}} \\
& \lesssim\left\|\chi_{(0,1)}(s) K\left(s, f_{0} ; A_{0}, A_{1}\right)\right\|_{\Phi_{0}}+\left\|\chi_{(1, \infty)}\right\|\left\|_{\Phi_{0}}\right\| f_{0} \|_{A_{0}} \\
& \approx\left\|\chi_{(0,1)}(s) K\left(s, f_{0} ; A_{0}, A_{1}\right)\right\|_{\Phi_{0}}+\left\|f_{0}\right\|_{A_{0}},
\end{aligned}
$$

where we have used (2.1). Next proceeding in the same way as in the proof of Theorem 3.3, we obtain

$$
\left\|f_{0}\right\|_{B_{0}} \lesssim\left\|\chi_{(0,1)}(s) K\left(s, f ; A_{0}, A_{1}\right)\right\|_{\Phi_{0}} .
$$

Also, we can show that

$$
\left\|f_{1}\right\|_{B_{1}} \lesssim\left\|\chi(1, \infty)(s) K\left(s, f ; A_{0}, A_{1}\right)\right\|_{\Phi_{1}},
$$

which, in view of $\left(\mathrm{C}_{5}\right)$, becomes

$$
\left\|f_{1}\right\|_{B_{1}} \lesssim\left\|\chi_{(0,1)}(s) s K\left(s^{-1}, f ; A_{0}, A_{1}\right)\right\|_{\Phi_{0}},
$$

which, combined with (3.4), yields

$$
\left\|f_{0}\right\|_{B_{0}}+\left\|f_{1}\right\|_{B_{1}} \lesssim\left\|\chi_{(0,1)}(s) K\left(s, f ; A_{0}, A_{1}\right)\right\|_{\Phi_{0}}+\left\|\chi_{(0,1)}(s) s K\left(s^{-1}, f ; A_{0}, A_{1}\right)\right\|_{\Phi_{0}},
$$

which, in view of Proposition 2.4, gives

$$
\left\|f_{0}\right\|_{B_{0}}+\left\|f_{1}\right\|_{B_{1}} \lesssim\|f\|_{B}
$$

from which the desired estimate $\|f\|_{B_{0}+B_{1}} \lesssim\|f\|_{B}$ follows. The proof of the theorem is finished.

Remark 3.11 Theorem 3.10, applied to the parameter spaces $\Phi_{0}$ and $\Phi_{1}$ given by (3.2) and (3.3), gives back (1.8) in [7], Theorem 1.1.

Corollary 3.12 Let $\left(A_{0}, A_{1}\right)$ be a compatible couple of normed spaces, and let $1 \leq p<\infty$. Then we have with equivalent norms

$$
\left(A_{0}, A_{1}\right)_{\{0\}, p}+\left(A_{0}, A_{1}\right)_{\{1\}, p}=\left(A_{0}+A_{1}, A_{0} \cap A_{1}\right)_{\{0\}, p} .
$$

Proof Apply Theorem 3.10 to the parameter spaces $\Phi_{0}$ and $\Phi_{1}$ given by (1.3) and (1.4).

Theorem 3.13 Let $\left(A_{0}, A_{1}\right)$ be a compatible couple of normed spaces, and assume that the parameter spaces $\Phi_{0}$ and $\Phi_{1}$ satisfy $\left(C_{1}\right)$. Then we have with equivalent norms

$$
\left(A_{0}, A_{0} \cap A_{1}\right)_{\Phi_{0}} \cap\left(A_{0}+A_{1}, A_{0} \cap A_{1}\right)_{\Phi_{1}}=\left(A_{0}, A_{0} \cap A_{1}\right)_{\Phi_{1}} .
$$


Proof Denote $B_{0}=\left(A_{0}, A_{0} \cap A_{1}\right)_{\Phi_{0}}, B_{1}=\left(A_{0}+A_{1}, A_{0} \cap A_{1}\right)_{\Phi_{1}}$, and $B=\left(A_{0}, A_{0} \cap A_{1}\right)_{\Phi_{1}}$. Let $f \in A_{0}$. The estimate $\|f\|_{B_{0}}+\|f\|_{B_{1}} \lesssim\|f\|_{B}$ follows thanks to the condition $\left(C_{1}\right)$ and the following simple inequality:

$$
K\left(t, f ; A_{0}+A_{1}, A_{0} \cap A_{1}\right) \leq K\left(t, f ; A_{0}, A_{0} \cap A_{1}\right), \quad t>0 .
$$

To derive the converse estimate, we apply Proposition 2.3 to obtain

$$
\|f\|_{B} \lesssim\left\|\chi_{(0,1)}(s) K\left(s, f ; A_{0}, A_{1}\right)\right\|_{\Phi_{1}}+\|f\|_{A_{0}} .
$$

Next, since $K\left(t, f ; A_{0}, A_{1}\right) / t$ is non-increasing in $t$, observe that

$$
\left\|\chi_{(0,1)}(s) K\left(s, f ; A_{0}, A_{0} \cap A_{1}\right)\right\|_{\Phi_{0}} \geq K\left(1, f ; A_{0}, A_{0} \cap A_{1}\right)\left\|s \chi_{(0,1)}(s)\right\|_{\Phi_{0}},
$$

noting $K\left(1, f ; A_{0}, A_{0} \cap A_{1}\right)=\|f\|_{A_{0}}$, we have

$$
\|f\|_{A_{0}} \lesssim\|f\|_{B_{0}} .
$$

By Proposition 2.4, we also have

$$
\left\|\chi_{(0,1)}(s) K\left(s, f ; A_{0}, A_{1}\right)\right\|_{\Phi_{1}} \lesssim\|f\|_{B_{1}} .
$$

Finally, combining (3.6), (3.7), and (3.8), we obtain $\|f\|_{B} \lesssim\|f\|_{B_{0}}+\|f\|_{B_{1}}$. The proof is finished.

Remark 3.14 By applying Theorem 3.13 to the parameter spaces $\Phi_{0}$ and $\Phi_{1}$ given by (3.2) and (3.3), we get back (1.9) in [7], Theorem 1.1, for $(\theta, p) \in \Gamma_{1}$.

Corollary 3.15 Let $\left(A_{0}, A_{1}\right)$ be a compatible couple of normed spaces, and let $1 \leq p<\infty$. Then we have with equivalent norms

$$
\left(A_{0}, A_{0} \cap A_{1}\right)_{\{0\}, p} \cap\left(A_{0}+A_{1}, A_{0} \cap A_{1}\right)_{\{1\}, p}=\left(A_{0}, A_{0} \cap A_{1}\right)_{\{1\}, p} .
$$

Proof Apply Theorem 3.13 to the parameter spaces $\Phi_{0}$ and $\Phi_{1}$ given by (1.3) and (1.4).

Theorem 3.16 Let $\left(A_{0}, A_{1}\right)$ be a compatible couple of normed spaces, and assume that the parameter spaces $\Phi_{0}$ and $\Phi_{1}$ satisfy $\left(C_{2}\right)$. Then we have with equivalent norms

$$
\left(A_{0}, A_{0} \cap A_{1}\right)_{\Phi_{0}} \cap\left(A_{0}+A_{1}, A_{0} \cap A_{1}\right)_{\Phi_{1}}=\left(A_{0}, A_{0} \cap A_{1}\right)_{\Phi_{0}} .
$$

Proof It will suffice to establish that $\left(A_{0}, A_{0} \cap A_{1}\right)_{\Phi_{0}} \hookrightarrow\left(A_{0}+A_{1}, A_{0} \cap A_{1}\right)_{\Phi_{1}}$. Let $f \in$ $\left(A_{0}, A_{0} \cap A_{1}\right)_{\Phi_{0}}$, then by (3.5) we have

$$
\left\|\chi_{(0,1)}(s) K\left(s, f ; A_{0}+A_{1}, A_{0} \cap A_{1}\right)\right\|_{\Phi_{1}} \leq\left\|\chi_{(0,1)}(s) K\left(s, f ; A_{0}, A_{0} \cap A_{1}\right)\right\|_{\Phi_{1}},
$$

consequently, in view of condition $\left(\mathrm{C}_{2}\right)$, we obtain

$$
\left\|\chi_{(0,1)}(s) K\left(s, f ; A_{0}+A_{1}, A_{0} \cap A_{1}\right)\right\|_{\Phi_{1}} \lesssim\left\|\chi_{(0,1)}(s) K\left(s, f ; A_{0}, A_{0} \cap A_{1}\right)\right\|_{\Phi_{0}},
$$

which concludes the proof. 
Remark 3.17 For $(\theta, p) \in \Gamma_{2}$, the result (1.9) in [7], Theorem 1.1, follows from Theorem 3.16, applied to the parameter spaces $\Phi_{0}$ and $\Phi_{1}$ given by (3.2) and (3.3).

Corollary 3.18 Let $\left(A_{0}, A_{1}\right)$ be a compatible couple of normed spaces, and let $1 \leq p<\infty$. Then we have with equivalent norms

$$
\left(A_{0}, A_{0} \cap A_{1}\right)_{\{1\}, p} \cap\left(A_{0}+A_{1}, A_{0} \cap A_{1}\right)_{\{0\}, p}=\left(A_{0}, A_{0} \cap A_{1}\right)_{\{1\}, p} .
$$

Proof Apply Theorem 3.16 to the parameter spaces $\Phi_{0}$ and $\Phi_{1}$ given by the norms

$$
\|g\|_{\Phi_{0}}=\sup _{0<s<1} \frac{|g(s)|}{s}+\left(\int_{1}^{\infty}\left(\frac{|g(s)|}{s}\right)^{p} \frac{d s}{s}\right)^{\frac{1}{p}}
$$

and

$$
\|g\|_{\Phi_{1}}=\left(\int_{0}^{1}|g(s)|^{p} \frac{d s}{s}\right)^{\frac{1}{p}}+\sup _{s>1}|g(s)| .
$$

Theorem 3.19 Let $\left(A_{0}, A_{1}\right)$ be a compatible couple of normed spaces, and assume that the parameter spaces $\Phi_{0}$ and $\Phi_{1}$ satisfy $\left(C_{2}\right)$. Then we have with equivalent norms

$$
\left(A_{0}+A_{1}, A_{1}\right)_{\Phi_{0}}+\left(A_{0}+A_{1}, A_{0} \cap A_{1}\right)_{\Phi_{1}}=\left(A_{0}+A_{1}, A_{1}\right)_{\Phi_{1}} .
$$

Proof Put $B_{0}=\left(A_{0}+A_{1}, A_{1}\right)_{\Phi_{0}}, B_{1}=\left(A_{0}+A_{1}, A_{0} \cap A_{1}\right)_{\Phi_{1}}$, and $B=\left(A_{0}+A_{1}, A_{1}\right)_{\Phi_{1}}$. Let $f \in$ $B_{0}+B_{1}$, and take an arbitrary decomposition $f=f_{0}+f_{1}$ with $f_{0} \in B_{0}$ and $f_{1} \in B_{1}$. Then by (2.4)

$$
\|f\|_{B} \lesssim\left\|\chi_{(0,1)}(s) K\left(s, f_{0} ; A_{0}+A_{1}, A_{1}\right)\right\|_{\Phi_{1}}+\left\|\chi_{(0,1)}(s) K\left(s, f_{1} ; A_{0}+A_{1}, A_{1}\right)\right\|_{\Phi_{1}},
$$

using condition $\left(C_{2}\right)$ and the fact that

$$
K\left(t, f_{1} ; A_{0}+A_{1}, A_{1}\right) \leq K\left(t, f_{1} ; A_{0}+A_{1}, A_{0} \cap A_{1}\right), \quad t>0,
$$

we obtain

$$
\|f\|_{B} \lesssim\left\|f_{0}\right\|_{B_{0}}+\left\|f_{1}\right\|_{B_{1}}
$$

whence, since $f=f_{0}+f_{1}$ is an arbitrary decomposition, we get $\|f\|_{B} \lesssim\|f\|_{B_{0}+B_{1}}$. For the converse estimate, let $f \in B$, and choose a particular decomposition $f=f_{0}+f_{1}$, with $f_{0} \in A_{0}$ and $f_{1} \in A_{1}$, satisfying (3.1). By Proposition 2.4,

$$
\left\|f_{0}\right\|_{B_{1}} \approx\left\|\chi_{(0,1)}(s) K\left(s, f_{0} ; A_{0}, A_{1}\right)\right\|_{\Phi_{1}}+\left\|\chi_{(0,1)}(s) s K\left(s^{-1}, f_{0} ; A_{0}, A_{1}\right)\right\|_{\Phi_{1}},
$$

using (2.1), we obtain

$$
\left\|f_{0}\right\|_{B_{1}} \lesssim\left\|\chi(0,1)(s) K\left(s, f_{0} ; A_{0}, A_{1}\right)\right\|_{\Phi_{1}}+\left\|f_{0}\right\|_{A_{0}}
$$


which, since $f_{0}=f-f_{1}$, gives

$$
\left\|f_{0}\right\|_{B_{1}} \lesssim\left\|\chi_{(0,1)}(s) K\left(s, f ; A_{0}, A_{1}\right)\right\|_{\Phi_{1}}+\left\|\chi_{(0,1)}(s) K\left(s, f_{1} ; A_{0}, A_{1}\right)\right\|_{\Phi_{1}}+\left\|f_{0}\right\|_{A_{0}},
$$

now using (2.2), it follows that

$$
\left\|f_{0}\right\|_{B_{1}} \lesssim\left\|\chi_{(0,1)}(s) K\left(s, f ; A_{0}, A_{1}\right)\right\|_{\Phi_{1}}+\left\|f_{1}\right\|_{A_{1}}+\left\|f_{0}\right\|_{A_{0}} .
$$

Using (2.2) also gives

$$
\begin{aligned}
\left\|f_{1}\right\|_{B_{0}} & \approx\left\|\chi_{(0,1)}(s) K\left(s, f_{1} ; A_{0}+A_{1}, A_{1}\right)\right\|_{\Phi_{0}} \\
& \lesssim\left\|f_{1}\right\|_{A_{1}}\left\|\chi_{(0,1)}(s)\right\|_{\Phi_{0}} \\
& \approx\left\|f_{1}\right\|_{A_{1}},
\end{aligned}
$$

which, together with (3.11), leads to

$$
\left\|f_{0}\right\|_{B_{1}}+\left\|f_{1}\right\|_{B_{0}} \lesssim\left\|\chi(0,1)(s) K\left(s, f ; A_{0}, A_{1}\right)\right\|_{\Phi_{1}}+\left\|f_{0}\right\|_{A_{0}}+\left\|f_{1}\right\|_{A_{1}},
$$

whence, in view of (3.1), it follows that

$$
\left\|f_{0}\right\|_{B_{1}}+\left\|f_{1}\right\|_{B_{0}} \lesssim\left\|\chi_{(0,1)}(s) K\left(s, f ; A_{0}, A_{1}\right)\right\|_{\Phi_{1}}+\|f\|_{A_{0}+A_{1}}
$$

according to 2.9 , we arrive at

$$
\left\|f_{0}\right\|_{B_{1}}+\left\|f_{1}\right\|_{B_{0}} \lesssim\left\|\chi_{(0,1)}(s) K\left(s, f ; A_{0}, A_{1}\right)\right\|_{\Phi_{1}},
$$

appealing to Proposition 2.2 yields

$$
\left\|f_{0}\right\|_{B_{1}}+\left\|f_{1}\right\|_{B_{0}} \lesssim\|f\|_{B}
$$

from which the desired estimate $\|f\|_{B_{0}+B_{1}} \lesssim\|f\|_{B}$ follows. The proof is complete.

Remark 3.20 We recover (1.10) in [7], Theorem 1.1, for $(\theta, p) \in \Gamma_{2}$, by an application of Theorem 3.19 to the parameter spaces $\Phi_{0}$ and $\Phi_{1}$ given by (3.2) and (3.3).

Corollary 3.21 Let $\left(A_{0}, A_{1}\right)$ be a compatible couple of normed spaces, and let $1 \leq p<\infty$. Then we have with equivalent norms

$$
\left(A_{0}+A_{1}, A_{1}\right)_{\{1\}, p}+\left(A_{0}+A_{1}, A_{0} \cap A_{1}\right)_{\{0\}, p}=\left(A_{0}+A_{1}, A_{1}\right)_{\{0\}, p} .
$$

Proof Apply Theorem 3.19 to the parameter spaces $\Phi_{0}$ and $\Phi_{1}$ given by (3.9) and (3.10).

Theorem 3.22 Let $\left(A_{0}, A_{1}\right)$ be a compatible couple of normed spaces, and assume that the parameter spaces $\Phi_{0}$ and $\Phi_{1}$ satisfy $\left(C_{1}\right)$. Then we have with equivalent norms

$$
\left(A_{0}+A_{1}, A_{1}\right)_{\Phi_{0}}+\left(A_{0}+A_{1}, A_{0} \cap A_{1}\right)_{\Phi_{1}}=\left(A_{0}+A_{1}, A_{1}\right)_{\Phi_{0}} .
$$


Proof It suffices to show that

$$
\left(A_{0}+A_{1}, A_{0} \cap A_{1}\right)_{\Phi_{1}} \hookrightarrow\left(A_{0}+A_{1}, A_{1}\right)_{\Phi_{0}} .
$$

Let $f \in\left(A_{0}+A_{1}, A_{0} \cap A_{1}\right)_{\Phi_{1}}$. Then, using condition $\left(C_{1}\right)$ and the elementary fact that

$$
K\left(t, f ; A_{0}+A_{1}, A_{1}\right) \leq K\left(t, f ; A_{0}+A_{1}, A_{0} \cap A_{1}\right), \quad t>0,
$$

we have

$$
\begin{aligned}
\left\|\chi_{(0,1)}(s) K\left(s, f ; A_{0}+A_{1}, A_{1}\right)\right\|_{\Phi_{0}} & \lesssim\left\|\chi_{(0,1)}(s) K\left(s, f ; A_{0}+A_{1}, A_{1}\right)\right\|_{\Phi_{1}} \\
& \leq\left\|\chi_{(0,1)}(s) K\left(s, f ; A_{0}+A_{1}, A_{0} \cap A_{1}\right)\right\|_{\Phi_{1}},
\end{aligned}
$$

which finishes the proof.

Remark 3.23 Theorem 3.22, applied to the parameter spaces $\Phi_{0}$ and $\Phi_{1}$ given by (3.2) and (3.3), gives back (1.10) in [7], Theorem 1.1, for $(\theta, p) \in \Gamma_{1}$.

Corollary 3.24 Let $\left(A_{0}, A_{1}\right)$ be a compatible couple of normed spaces, and let $1 \leq p<\infty$. Then we have with equivalent norms

$$
\left(A_{0}+A_{1}, A_{1}\right)_{\{0\}, p}+\left(A_{0}+A_{1}, A_{0} \cap A_{1}\right)_{\{1\}, p}=\left(A_{0}+A_{1}, A_{1}\right)_{\{0\}, p} .
$$

Proof Apply Theorem 3.22 to the parameter spaces $\Phi_{0}$ and $\Phi_{1}$ given by (1.3) and (1.4).

Theorem 3.25 Let $\left(A_{0}, A_{1}\right)$ be a compatible couple of normed spaces, and assume that the parameter spaces $\Phi_{0}$ and $\Phi_{1}$ satisfy $\left(C_{1}\right)$. Then we have with equivalent norms

$$
\left(A_{0}, A_{0} \cap A_{1}\right)_{\Phi_{0}}+\left(A_{0}+A_{1}, A_{0} \cap A_{1}\right)_{\Phi_{1}}=\left(A_{0}, A_{1}\right)_{\Psi}
$$

where

$$
\|g\|_{\Psi}=\left\|\chi_{(0,1)}(s) g(s)\right\|_{\Phi_{0}}+\left\|\chi_{(0,1)}(s) \operatorname{sg}(1 / s)\right\|_{\Phi_{1}} .
$$

Proof Set $B_{0}=\left(A_{0}, A_{0} \cap A_{1}\right)_{\Phi_{0}}$ and $B_{1}=\left(A_{0}+A_{1}, A_{0} \cap A_{1}\right)_{\Phi_{1}}$. Let $f \in B_{0}+B_{1}$, and write $f=f_{0}+f_{1}$ with $f_{0} \in B_{0}$ and $f_{1} \in B_{1}$. Making use of (2.4), we have

$$
\|f\|_{\left(A_{0}, A_{1}\right)_{\Psi}} \lesssim I_{1}+I_{2}
$$

where

$$
I_{1}=\left\|\chi_{(0,1)}(s) K\left(s, f_{0} ; A_{0}, A_{1}\right)\right\|_{\Phi_{0}}+\left\|\chi_{(0,1)}(s) K\left(s, f_{1} ; A_{0}, A_{1}\right)\right\|_{\Phi_{0}}
$$

and

$$
I_{2}=\left\|\chi_{(0,1)}(s) s K\left(s^{-1}, f_{0} ; A_{0}, A_{1}\right)\right\|_{\Phi_{1}}+\left\|\chi_{(0,1)}(s) s K\left(s^{-1}, f_{1} ; A_{0}, A_{1}\right)\right\|_{\Phi_{1}} .
$$


The condition $\left(C_{1}\right)$, along with the following simple inequality:

$$
K\left(t, f_{0} ; A_{0}, A_{1}\right) \leq K\left(t, f_{0} ; A_{0}, A_{0} \cap A_{1}\right), \quad t>0,
$$

implies that

$$
I_{1} \lesssim\left\|\chi_{(0,1)}(s) K\left(s, f_{0} ; A_{0}, A_{0} \cap A_{1}\right)\right\|_{\Phi_{0}}+\left\|\chi_{(0,1)}(s) K\left(s, f_{1} ; A_{0}, A_{1}\right)\right\|_{\Phi_{1}} .
$$

Next we observe that $f_{0} \in A_{0}$ as $B_{0} \subset A_{0}$. Therefore, we can apply (2.1) to arrive at

$$
I_{2} \lesssim\left\|f_{0}\right\|_{A_{0}}+\left\|\chi \chi_{(0,1)}(s) s K\left(s^{-1}, f_{1} ; A_{0}, A_{1}\right)\right\|_{\Phi_{1}} .
$$

The proof of the estimate

$$
\left\|f_{0}\right\|_{A_{0}} \lesssim\left\|\chi_{(0,1)}(s) K\left(s, f_{0} ; A_{0}, A_{0} \cap A_{1}\right)\right\|_{\Phi_{0}}
$$

is the same as that of (3.7). Finally, inserting estimates (3.13) and (3.14) in (3.12) and then using (3.15) and Proposition 2.4, we get

$$
\|f\|_{\left(A_{0}, A_{1}\right)_{\Psi}} \lesssim\left\|f_{0}\right\|_{B_{0}}+\left\|f_{1}\right\|_{B_{1}}
$$

which gives the estimate $\|f\|_{\left(A_{0}, A_{1}\right)_{\Psi}} \lesssim\|f\|_{B_{0}+B_{1}}$. In order to prove the other estimate, we take $f \in\left(A_{0}, A_{1}\right)_{\Psi}$, and select a particular decomposition $f=f_{0}+f_{1}$, with $f_{0} \in A_{0}$ and $f_{1} \in A_{1}$, satisfying condition (3.1). Then proceeding in the same way as in the proof of Theorem 3.3, we obtain

$$
\left\|f_{0}\right\|_{B_{0}} \lesssim\left\|\chi_{(0,1)}(s) K\left(s, f ; A_{0}, A_{1}\right)\right\|_{\Phi_{0}} .
$$

Also, we have

$$
\left\|f_{1}\right\|_{B_{1}} \lesssim\left\|\chi_{(0,1)}(s) s K\left(s^{-1}, f ; A_{0}, A_{1}\right)\right\|_{\Phi_{1}} .
$$

Therefore, these estimates, along with the definition of $\Psi$, imply that

$$
\left\|f_{0}\right\|_{B_{0}}+\left\|f_{1}\right\|_{B_{1}} \lesssim\|f\|_{\left(A_{0}, A_{1}\right)_{\Psi}}
$$

whence we get $\|f\|_{B_{0}+B_{1}} \lesssim\|f\|_{\left(A_{0}, A_{1}\right)_{\Psi}}$. The proof is finished.

Remark 3.26 Take $\Phi_{0}$ and $\Phi_{1}$ to be given by (3.2) and (3.3), then we see that $\Psi=\Phi_{0}$. Thus, we recover the result (1.11) in [7], Theorem 1.1, for $(\theta, p) \in \Gamma_{1}$. Since the case when $(\theta, p) \in \Gamma_{2}$ follows from the case when $(\theta, p) \in \Gamma_{1}$, Theorem 3.25 provides a generalization of the assertion (1.11) in [7], Theorem 1.1.

Corollary 3.27 Let $\left(A_{0}, A_{1}\right)$ be a compatible couple of normed spaces, and let $1 \leq p<\infty$. Then we have with equivalent norms

$$
\left(A_{0}, A_{0} \cap A_{1}\right)_{\{0\}, p}+\left(A_{0}+A_{1}, A_{0} \cap A_{1}\right)_{\{1\}, p}=\left(A_{0}, A_{1}\right)_{\{0\}, p} .
$$


Proof Apply Theorem 3.25 to the parameter spaces $\Phi_{0}$ and $\Phi_{1}$ given by (1.3) and (1.4).

Theorem 3.28 Let $\left(A_{0}, A_{1}\right)$ be a compatible couple of normed spaces, and assume that the parameter spaces $\Phi_{0}$ and $\Phi_{1}$ satisfy $\left(C_{2}\right)$. Then we have with equivalent norms

$$
\left(A_{0}+A_{1}, A_{0}\right)_{\Phi_{0}} \cap\left(A_{0}+A_{1}, A_{0} \cap A_{1}\right)_{\Phi_{1}}=\left(A_{0}, A_{1}\right)_{\Psi}
$$

where

$$
\|g\|_{\Psi}=\left\|\chi_{(0,1)}(s) g(s)\right\|_{\Phi_{1}}+\left\|\chi_{(0,1)}(s) \operatorname{sg}(1 / s)\right\|_{\Phi_{0}} .
$$

Proof Set $B_{0}=\left(A_{0}+A_{1}, A_{0}\right)_{\Phi_{0}}$ and $B_{1}=\left(A_{0}+A_{1}, A_{0} \cap A_{1}\right)_{\Phi_{1}}$. Let $f \in A_{0}+A_{1}$. Applying Proposition 2.2 to the compatible couple $\left(A_{1}, A_{0}\right)$, we get

$$
\|f\|_{B_{0}} \approx\left\|\chi_{(0,1)}(s) K\left(s, f ; A_{1}, A_{0}\right)\right\|_{\Phi_{0}},
$$

using (2.3), we have

$$
\|f\|_{B_{0}} \approx\left\|\chi_{(0,1)}(s) s K\left(s^{-1}, f ; A_{0}, A_{1}\right)\right\|_{\Phi_{0}} .
$$

By Proposition 2.4,

$$
\|f\|_{B_{1}} \approx\left\|\chi_{(0,1)}(s) K\left(s, f ; A_{0}, A_{1}\right)\right\|_{\Phi_{1}}+\left\|\chi_{(0,1)}(s) s K\left(s^{-1}, f ; A_{0}, A_{1}\right)\right\|_{\Phi_{1}},
$$

combining this with (3.16) and making use of $\left(\mathrm{C}_{2}\right)$, we arrive at

$$
\|f\|_{B_{0}}+\|f\|_{B_{1}} \approx\|f\|_{\left(A_{0}, A_{1}\right)_{\Psi}},
$$

which completes the proof.

Remark 3.29 Theorem 3.28 generalizes the result (1.12) in [7], Theorem 1.1.

Corollary 3.30 Let $\left(A_{0}, A_{1}\right)$ be a compatible couple of normed spaces, and let $1 \leq p<\infty$. Then we have with equivalent norms

$$
\left(A_{0}+A_{1}, A_{0}\right)_{\{1\}, p}+\left(A_{0}+A_{1}, A_{0} \cap A_{1}\right)_{\{0\}, p}=\left(A_{0}, A_{1}\right)_{\{0\}, p} .
$$

Proof Apply Theorem 3.28 to the parameter spaces $\Phi_{0}$ and $\Phi_{1}$ given by (3.9) and (3.10).

Competing interests

The authors declare that they have no competing interests.

Authors' contributions

All authors have equally contributed toward the article. All authors read and approved the final manuscript.

Received: 3 September 2016 Accepted: 15 November 2016 Published online: 25 November 2016 
References

1. Brudnyi, YA, Krugljak, NY: Interpolation Functors and Interpolation Spaces, vol. 1. North-Holland, Amsterdam (1991)

2. Bergh, J, Löfström, J: Interpolation Spaces. An Introduction. Springer, New York (1976)

3. Bennett, C, Sharpley, R: Interpolation of Operators. Academic Press, New York (1988)

4. Maligranda, L: The K-functional for symmetric spaces. In: Interpolation Spaces and Allied Topics in Analysis. Lecture Notes in Math., vol. 1070, pp. 170-182. Springer, Berlin (1984)

5. Maligranda, L: Interpolation between sum and intersection of Banach spaces. J. Approx. Theory 47, $42-53$ (1986)

6. Persson, LE: Interpolation with a parameter function. Math. Scand. 59, 199-222 (1986)

7. Haase, M: Identification of some real interpolation spaces. Proc. Am. Math. Soc. 134, 2349-2358 (2006)

8. Cobos, F, Fernández-Cabrera, LM, Silvestre, P: New limiting real interpolation methods and their connection with the methods associated to the unit square. Math. Nachr. 286, 569-578 (2013)

\section{Submit your manuscript to a SpringerOpen ${ }^{\circ}$} journal and benefit from:

- Convenient online submission

- Rigorous peer review

- Immediate publication on acceptance

- Open access: articles freely available online

- High visibility within the field

- Retaining the copyright to your article 